\title{
Characteristic Parameters of a Wide Cluster in a Higher-Order Traffic Flow Model *
}

\author{
ZHANG Peng $^{1} \quad$ WONG S.C. ${ }^{2 \dagger}$ DAI Shi-Qiang ${ }^{1}$ \\ 1. Shanghai Institute of Applied Mathematics and Mechanics, Shanghai University, \\ Shanghai 200072, China \\ 2. Department of Civil Engineering, The University of Hong Kong, \\ Hong Kong SAR, China
}

\begin{abstract}
Nonlinear weak solution theory is applied to determine the parameters of a wide cluster in an 'anisotropic' higher-order traffic flow model. These parameters are the maximal and minimal densities and the traveling wave speed in the solution structure. Numerical experiments show that the convergent simulation results are in good agreement with those that are obtained from the analytical expressions.
\end{abstract}

In the macroscopic description that considers traffic vehicles as a continuum fluid, the higher-order models receive much attention because of their ability to reproduce stop-and-go waves or clusters. These models are mostly of the P-W [1-2] or K-K type [3-4], for which many properties of clusters have been investigated. We remark that the formation of a cluster embodies a mechanism of physical metastability in which traffic flow may evolve from one steady state into another stable state under a small perturbation [5-10]. These models are 'isotropic', which means that a perturbation propagates both upstream and downstream from the perturbed media, because the two characteristic speeds $\lambda_{1}=v-c$ and $\lambda_{2}=v+c$ (where the sound speed $c>0$ ) are respectively smaller and greater than the motion speed $v$. The propagation that corresponds to $\lambda_{2}$ is regarded to be physically unsound in traffic flow, because vehicles are influenced by the traffic upstream [11]. As a consequence, some 'anisotropic' higher-order models have been developed in recent years, which are distinguished by the two characteristic speeds $\lambda_{1}=v-c$ and $\lambda_{2}=v$ [12-15].

In this paper, we apply the weak solution theory of hyperbolic conservation laws to higher-order traffic flow models. Accordingly, we are able to confirm whether or not the solution structure of a wide cluster exists, and analytically determine the maximal and minimal densities and traveling wave speed of such a solution. In this context, we choose the model in Ref.[13] as an example, because it has an elegant expression of acceleration by which a conservation form can be easily defined.

With the density notated as $\rho(x, t)$, the velocity as $v(x, t)$, and the flow as $q=\rho v$ in location $x$ and at time $t$, traffic flow can be viewed as a continuum, the mass conservation of which reads:

$$
\frac{\partial \rho}{\partial t}+\frac{\partial \rho v}{\partial x}=0
$$

We rewrite the acceleration equation in Ref.[13] in the following conservation form:

$$
\frac{\partial v}{\partial t}+\frac{\partial\left(v^{2} / 2-c v\right)}{\partial x}=\frac{v_{e}(\rho)-v}{\tau}
$$

${ }^{*}$ The work described in this paper was jointly supported by grants from the Hong Kong Research Grants Council of the Hong Kong Special Administrative Region, China (Project No. HKU 7031/02E) and the National Natural Science Foundation of China (Grant No. 10532060, 10472064). The authors would like to thank Professor C.-W.Shu, Chairman of the Division of Applied Mathematics at Brown University, for his helpful and constructive comments on our work.

${ }^{\dagger}$ Corresponding author. E-mail: hhecwsc@hkucc.hku.hk, Tel:+852-2859-1964, Fax:+852-2559-5337. 
where the sound speed $c$ is constant. Therefore, Eqs. (1) and (2) constitute a complete conservation system for a weak solution. Furthermore, we apply a non-convex velocity-density relation

$$
v_{e}(\rho)=v_{f}\left[\left(1+\exp \left(\frac{\rho / \rho_{m}-0.25}{0.08}\right)\right)^{-1}-\left(1+\exp \left(\frac{0.75}{0.08}\right)\right)^{-1}\right],
$$

where $v_{f}$ is the free-flow velocity and $\rho_{m}$ is the maximal density. This function is similar to that in Ref.[3-4]. Other non-convex functions that are given in Ref.[8] are also applicable to our discussion.

In the following, we investigate the existence of a traveling wave solution to Eqs. (1) and (2), that represents a wide cluster in the density-space plane with determined maximal and minimal densities and traveling wave speed. Dimensionless variables or functions are adopted in all of the illustrations and subsequent discussions, and each is distinguished from the original variable by an overhead bar such that the density is scaled by $\rho_{m}$ (e.g., $\bar{\rho}=\rho / \rho_{m}$ ), the velocity by $v_{f}$, the flow by $\rho_{m} v_{f}$, and the length by $L$, where $L$ is the computational length.

The traveling wave solution is a steady state through the transformation $X=x-$ at. Taking three constant density segments, which are represented by $\rho=\rho_{1}, \rho=\rho_{2}$, and $\rho=\rho_{1}$ in $X-\rho$ coordinates (Fig.1(a)) and which correspond to the two phase states $\left(\rho_{1}, q_{e}\left(\rho_{1}\right)\right)$ and $\left(\rho_{2}, q_{e}\left(\rho_{2}\right)\right)$ in the fundamental diagram (Fig.1(b)), we study the complete traveling wave solution for this problem. Two adjacent constant states can be connected by a smooth piece of curve $\rho=\rho(X)$, which is determined by Eqs. (1) and (2). Alternatively, the states can be separated by a discontinuity that is determined by the Rankine-Hugoniot jump conditions according to the conservation equations.

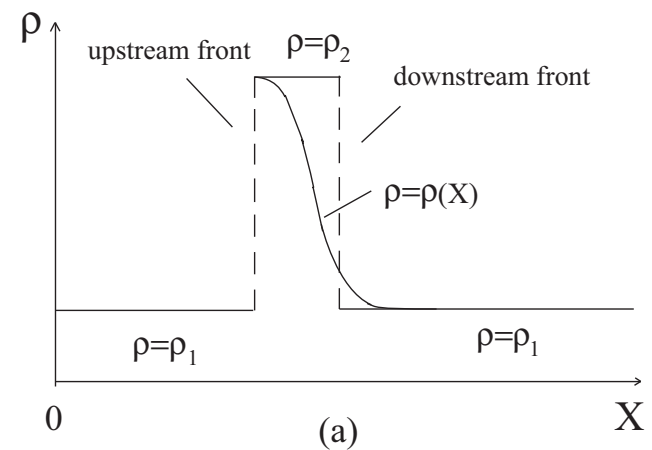

(a)

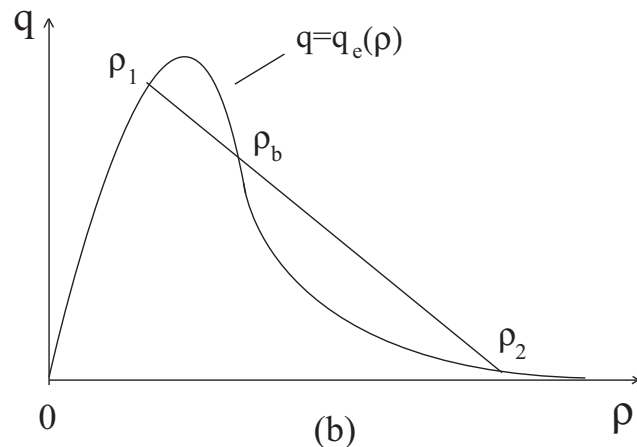

(b)

Fig.1 Identification of a cluster as a traveling wave solution, through connection by a smooth curve, or separation by a discontinuity of two adjacent constant states, (a) steady density distribution in the transformed coordinates; (b) phase states $(\rho, q)$ and the fundamental diagram $q=q_{e}(\rho) \equiv \rho v_{e}(\rho)$.

We first rewrite the solution as $\rho=\rho(X)$ and $v=v(X)$ in Eqs. (1) and (2), which yields

$$
\frac{d \rho}{d X}=g(\rho, v) \frac{q_{e}(\rho)-q}{c \rho+A}, \quad q=a \rho-A
$$

where $g=\rho[\tau(v-a)]^{-1}$ and $A$ is the integration constant (see Ref.[2] for a similar derivation). The last equality of (3) indicates that the transitional curve corresponds to a segment of the straight line between the $\rho_{1}$ and $\rho_{2}$ phase states in Fig.1(b). The slope $a$ and the constant $A$ are accordingly determined to be

$$
a=\frac{q_{e}\left(\rho_{2}\right)-q_{e}\left(\rho_{1}\right)}{\rho_{2}-\rho_{1}}, \quad A=\rho_{1} \rho_{2} \frac{v_{e}\left(\rho_{2}\right)-v_{e}\left(\rho_{1}\right)}{\rho_{2}-\rho_{1}} .
$$

It is obvious that $g(\rho, v)>0$ and $A<0$. Moreover, a larger $\tau$ gives a smaller slope $d \rho / d X$, and thus a smoother transitional layer. Also note that the velocity $v$ of the state in Fig.1(b) is the slope of the straight line that links this state to the origin.

For the downstream front (Fig.1(a)), we must have $d \rho / d X<0$. Assume that $\left(\rho_{b}, q_{e}\left(\rho_{b}\right)\right)$ is the intersection of the curve $q=q_{e}(\rho)$ and the segment $\rho_{1} \rho_{2}$ (Fig.1(b)) with $q_{e}\left(\rho_{b}\right)=a \rho_{b}-A$. Then 
$q_{e}(\rho)-q$ is positive in the $\rho_{1} \rho_{b}$ piece, but negative in the $\rho_{b} \rho_{2}$ piece. This means that $c \rho+A$ must be negative in $\rho_{1} \rho_{b}$ but positive in $\rho_{b} \rho_{2}$, and thus by continuity we must have $c \rho_{b}+A=0$. The equalities are therefore rewritten as

$$
q_{e}\left(\rho_{b}\right)=a \rho_{b}-A, \quad c \rho_{b}+A=0
$$

For the upstream front (Fig.1(a)), it is required that $d \rho / d X>0$ and consequently that $c \rho+A$ be positive in $\rho_{1} \rho_{b}$ but negative in $\rho_{b} \rho_{2}$. This is contrary to the scenario in the downstream front and the fact that $c \rho+A$ is increasing in $\rho$. Thus, we must introduce a discontinuity that propagates at the same speed $a$ to separate the two adjacent constant states. For a conservative system with a source term that is a bounded function of the solution variables, the Rankine-Hugoniot jump conditions can be applied, which take the same form as the conditions in the corresponding homogeneous system (see Ref. [2,10,16-23] to learn how the conditions are applied in traffic flow problems). This application also gives the first equality of (4) and

$$
a=\frac{1}{2}\left(v_{e}\left(\rho_{1}\right)+v_{e}\left(\rho_{2}\right)\right)-c .
$$

We now have five unknowns, $\rho_{1}, \rho_{2}, \rho_{b}, a$, and $A$, which can be uniquely determined from the five equations in (4)-(6). Using the scaled dimensionless variables, only $\bar{c}$ (but not the parameters $v_{f}$ and $\rho_{m}$ ) in a certain range is needed to compute $\bar{\rho}_{1}, \bar{\rho}_{2}$, etc. (see Table 1 ). These approximate values are shown in Table 1 for $\bar{c}=0.45$ to $\bar{c}=0.6$ in increments of 0.01 . Note that the sound speed $\bar{c}$ that is smaller than 0.51 is not physically valid, because the corresponding $\bar{\rho}_{2}$ is greater than 1 .

Table 1: The minimal and maximal densities $\bar{\rho}_{1}$ and $\bar{\rho}_{2}$ of a cluster as determined by Eqs. (4)-(6) for a range of $\bar{c}$, along with the transitional density $\bar{\rho}_{b}$ and traveling speed $\bar{a}$. Two critical densities, $\bar{\rho}_{c_{1}}$ and $\bar{\rho}_{c_{2}}$, for a linearly stable range $\bar{\rho}_{0} \in\left[\bar{\rho}_{c_{1}}, \bar{\rho}_{c_{2}}\right]$ are also given.

\begin{tabular}{|c|c|c|c|c|c|c|}
\hline $\bar{c}$ & $\bar{\rho}_{1}$ & $\bar{\rho}_{2}$ & $\bar{\rho}_{b}$ & $\bar{a}$ & $\bar{\rho}_{c_{1}}$ & $\bar{\rho}_{c_{2}}$ \\
\hline 0.45 & 0.15604 & 1.90853 & 0.28849 & -0.06811 & 0.17654 & 0.43380 \\
\hline 0.46 & 0.15878 & 1.64171 & 0.28955 & -0.08216 & 0.17855 & 0.43101 \\
\hline 0.47 & 0.16152 & 1.44841 & 0.29063 & -0.09439 & 0.18056 & 0.42824 \\
\hline 0.48 & 0.16427 & 1.30201 & 0.29174 & -0.10764 & 0.18255 & 0.42551 \\
\hline 0.49 & 0.16704 & 1.18733 & 0.29287 & -0.12094 & 0.18454 & 0.42279 \\
\hline 0.50 & 0.16982 & 1.09505 & 0.29403 & -0.13431 & 0.18653 & 0.42010 \\
\hline 0.51 & 0.17261 & 1.01913 & 0.29522 & -0.14776 & 0.18851 & 0.41743 \\
\hline 0.52 & 0.17543 & 0.95553 & 0.29644 & -0.16126 & 0.19050 & 0.41477 \\
\hline 0.53 & 0.17828 & 0.90141 & 0.29768 & -0.17482 & 0.19248 & 0.41213 \\
\hline 0.54 & 0.18115 & 0.85474 & 0.29894 & -0.18843 & 0.19447 & 0.40950 \\
\hline 0.55 & 0.18406 & 0.81399 & 0.30022 & -0.20208 & 0.19646 & 0.40688 \\
\hline 0.56 & 0.18700 & 0.77802 & 0.30153 & -0.21576 & 0.19845 & 0.40427 \\
\hline 0.57 & 0.19899 & 0.74597 & 0.30284 & -0.22946 & 0.20046 & 0.40167 \\
\hline 0.58 & 0.19302 & 0.71715 & 0.30417 & -0.24318 & 0.20247 & 0.39907 \\
\hline 0.59 & 0.19610 & 0.69103 & 0.30551 & -0.25691 & 0.20449 & 0.39647 \\
\hline 0.60 & 0.19925 & 0.66717 & 0.30686 & -0.27064 & 0.20653 & 0.39387 \\
\hline
\end{tabular}

To generate a wide cluster through numerical simulation, we take the following initial and boundary conditions

$$
\rho(x, 0)=\rho_{0}+\Delta \rho_{0}\left\{\cosh ^{-2}\left[\frac{160}{L}\left(x-\frac{7 L}{16}\right)\right]-\frac{1}{4} \cosh ^{-2}\left[\frac{40}{L}\left(x-\frac{15 L}{32}\right)\right]\right\}
$$




$$
v(x, 0)=v_{e}(\rho(x, 0)) ; \quad \rho(0, t)=\rho(L, t), \quad v(0, t)=v(L, t),
$$

which are also similar to those in Ref.[3-4]. Here, the last term in $\rho(x, 0)$ represents a perturbation to a stationary condition with a constant density $\rho=\rho_{0}$ that may be unstable and evolve into stopand-go waves. The parameters are taken as $\rho_{m}=0.15 v e h . / m, v_{f}=30 \mathrm{~m} / \mathrm{s}, L=20000 \mathrm{~m}, \bar{\rho}_{0}=0.22$, and $\Delta \bar{\rho}_{0}=0.08$.

Fig. 2 shows that the values of the maximal and minimal densities and the traveling wave speed agree very well with those in Table 1 . With sufficiently small grid lengths in the numerical simulation, this is also true for the comparison to which other values of the sound speed $\bar{c}$ are applied. Note that only one state in each length of 20 grids is shown in Figs. 2(b),(d), and (f).
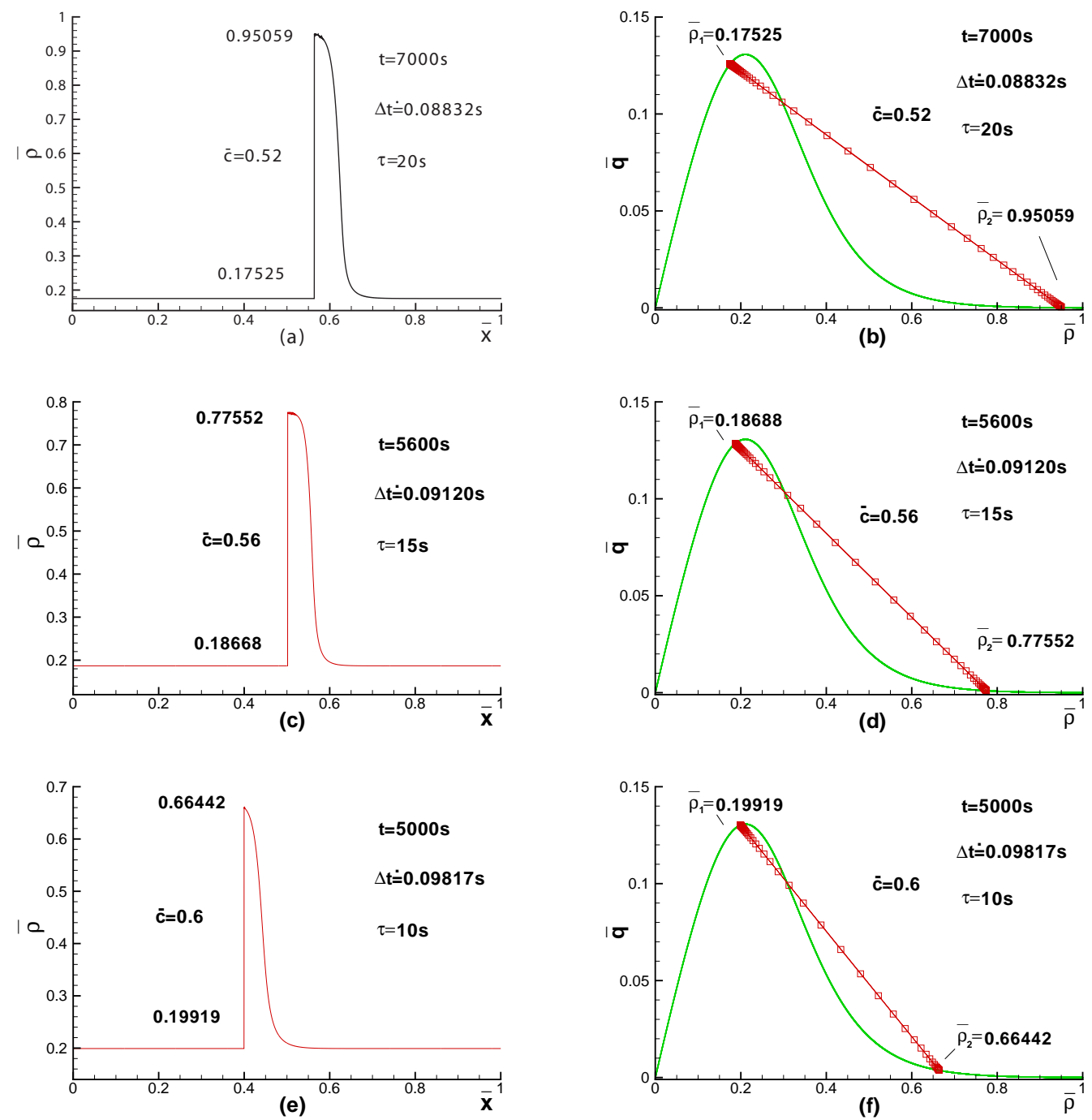

Fig.2 Fully evolved cluster through numerical simulation with a sufficiently large grid number $N=10^{4}$, (a), (c), and (e): distribution of the density; (b), (d), and (f): density-flow states and the fundamental diagram.

Two critical densities, $\bar{\rho}_{c_{1}}$ and $\bar{\rho}_{c_{2}}$, are also given in Table 1 . They are related to the linearized equation

$$
\xi_{t}+\lambda_{0} \xi_{x}=\tau\left(\lambda_{2}-\lambda_{0}\right)\left(\lambda_{0}-\lambda_{1}\right) \xi_{x x},
$$

where $\xi$ represents the perturbation as a density of the constant states $\rho=\rho_{0}$ and $v=v_{0} \equiv v_{e}\left(\rho_{0}\right)$, $\lambda_{0}=v_{e}\left(\rho_{0}\right)+\rho_{0} v_{e}^{\prime}\left(\rho_{0}\right)$ is the kinematic wave speed, and $\lambda_{1}=v_{0}$ and $\lambda_{2}=v_{0}-c$ are the two characteristic speeds at $\left(\rho_{0}, v_{0}\right)$ (see Ref.[2] for a detailed derivation). A traffic state $\left(\rho_{0}, v_{0}\right)$ is 
linearly stable only with a positive viscosity in Eq.(8), that is, $c+\rho_{0} v_{e}^{\prime}\left(\rho_{0}\right)>0$. This is equivalent to $\rho_{c_{1}} \leq \rho_{0} \leq \rho_{c_{2}}$. According to Eq.(8), a larger $\tau$ suggests more viscosity to smooth out the transitional layer. Note that in Table $1 \overline{\rho_{1}}$ and $\overline{\rho_{2}}$ fall outside the interval $\left[\overline{\rho_{c_{1}}}, \overline{\rho_{c_{1}}}\right]$, which explains why the equilibrium states of $\rho=\rho_{1}$ and $\rho=\rho_{2}$ (the corresponding velocities that satisfy $v_{1}=v_{e}\left(\rho_{1}\right)$ and $\left.v_{2}=v_{e}\left(\rho_{2}\right)\right)$ in the cluster structure are stable.

In summary, we prove that a wide cluster is truly a traveling wave solution to Eqs. (1) and (2). The characteristic parameters of the solution are analytically determined and are in good agreement with the numerical results. The theory and the procedure can also be applied to study other higher-order traffic flow models, provided that their conservation forms can be defined.

\section{References}

[1] Payne H J 1971 Models of Freeway Traffic and Control ed Bekey A G (La Jola: Mathematical Models of Public Systems, Simulation Council Proc.) vol 1 p 51-61

[2] Whitham G B 1974 Linear and Nonlinear Waves (New York: John Wiley and Sons)

[3] Kerner B S and Konhauser P 1993 Phys. Rev. E 48 2335-2338

[4] Kerner B S and Konhauser P 1994 Phys. Rev. E 50 54-83

[5] Kerner B S and Rehborn H 1997 Phys. Rev. Letters 79 4030-4033

[6] Jin W L and Zhang H M 2003 Transp. Res. B 37 207-223

[7] Li T and Liu H L 2005 Comm. Math. Sci. 3 101-118

[8] Li T 2005 Physica D 207 41-51

[9] Helbing D 2001 Reviews of Modern Physics 73 1067-1141

[10] Zhang P, Liu R X and Wong S C 2005 Phys. Rev. E 71056704

[11] Daganzo C F 1995 Transpn. Res. B 29 277-286

[12] Aw A and Rascle M 2000 SIAM J. Appl. Math. 60 916-938

[13] Jiang R, Wu Q S and Zhu Z J 2002 Transp. Res. B 36 405-419

[14] Xue Y and Dai S Q 2003 Phys. Rev. E 68066123

[15] Zhang H M 2002 Transp. Res. B 36 275-290

[16] LeVeque R J 2002 Finite Volume Methods for Hyperbolic Systems (New York: Cambridge University Press)

[17] Zhang P and Liu R X 2003 J. Comput. Appl. Math. 156 1-21

[18] Zhang P and Liu R X 2005 J. Comput. Appl. Math. 176 105-129

[19] Zhang P and Liu R X 2003 Numerical Methods for Partial Differential Equations 21 80-88

[20] Wong S C and Wong G C K 2002 Transp, Res. B 36 683-706.

[21] Wong G C K and Wong S C 2002 Transp, Res. A 36 827-841.

[22] Zhang P, Liu R X, Wong S C and Dai S Q European Journal of Applied Mathematics, in press.

[23] Zhang P, Wong S C and Shu C W 2006 J. of Computational Physics 212 739-756 